\title{
Medical Sample Classifier Design Using Fuzzy Cerebellar Model Neural Networks
}

\author{
Hsin-Yi Li ${ }^{1}$, Rong-Guan Yeh ${ }^{2}$, Yu-Che Lin ${ }^{3}$, Lo-Yi Lin ${ }^{4}$, \\ Jing Zhao ${ }^{5}$, Chih-Min Lin ${ }^{6, *}$, Imre J. Rudas ${ }^{7}$ \\ 1 \\ Information and Communications Research Division, National Chung-Shan
} Institute of Science \& Technology, Taoyuan 320, Taiwan,

s988506@mail.yzu.edu.tw

2

School of Mechanical \& Electronic Engineering, Sanming University, Sanming 365004, China, styeh@ @aturn.yzu.edu.tw

3

Information and Communications Research Division, National Chung-Shan

Institute of Science \& Technology, Taoyuan 320, Taiwan,

s1004603@mail.yzu.edu.tw

4

School of Medicine, Taipei Medical University, Taipei 100, Taiwan, zoelin56@hotmail.com

5

School of Electrical Engineering \& Automation, Xiamen University of Technology, Xiamen 361000, China; and Department of Electrical Engineering, Yuan Ze University, Tao-Yuan 320, Taiwan, jzhao@xmut.edu.cn

$6^{*}$

Corresponding author, Department of Electrical Engineering, Yuan Ze

University, Chung-Li, Taoyuan 320, Taiwan; and School of Information Science and Engineering, Xiamen University, Xiamen 361000, China,

cml@saturn.yzu.edu.tw

${ }^{7}$ Óbuda University, Bécsi út 96/b, H-1034 Budapest, Hungary, e-mail: rudas@uniobuda.hu

Abstract: This study designs a stable and convergent adaptive fuzzy cerebellar model articulation classifier for the identification of medical samples. Research on medical diagnosis is important and complex. Uncertainty is unavoidable in medical diagnosis. The symptoms are one of the uncertainties of an illness and may or may not occur as a result of the disease. There is an uncertain relationship between symptoms and disease. Diagnostic accuracy can be improved using data forecasting techniques. This study proposes a generalized fuzzy neural network, called a fuzzy cerebellar model articulation controller (FCMAC). It is an expansion of a fuzzy neural network and it will be shown that the traditional fuzzy neural network controller is a special case of this FCMAC. This expansion type fuzzy neural network has a greater ability to be generalized, has greater learning 
ability and a greater capacity to approximate than a traditional fuzzy neural network. The steepest descent method has a fast convergent characteristic, so it is used to derive the adaptive law for the adaptive fuzzy cerebellar model classifier. The optimal learning rate is also derived to achieve the fastest convergence for the FCMAC, in order to allow fast and accurate identification. The simulation results demonstrate the effectiveness of the proposed FCMAC classifier.

Keywords: cerebellar model articulation controller; fuzzy rule; classifier; diseases diagnosis

\section{Introduction}

Recently, neural networks have been widely used for system identification and control problems [1-4]. The most prominent feature of a neural network is its ability to approximate.

The Cerebellar Model Articulation Controller (CMAC), was first proposed by Marr [5] in 1969. In 1975, Albus published two articles on the CMAC, based on the cerebellar cortex model of Marr [6, 7], including the mathematical algorithms and memory storage methods for a CMAC. A CMAC is a neural network that behaves similarly to the human cerebellum. It is classified as a non-fully connected perceptual machine type network. A CMAC can be used to imitate an object. The imitating process can be divided into the learning and recalling processes. The CMAC can repeat the learning process and correct the memory data until the desired results are achieved. In contrast with other neural networks, the CMAC does not use abstruse mathematical calculation. It has the advantage of a simple structure, easy operation, fast learning convergence speed, an ability to classify regions and it is easy to implement, so it is suitable for application to online learning systems.

Previously, CMACs have been the subject of many studies by researchers in various fields and many studies have verified that CMACs perform better than neural networks in many applications [8,9]. CMACs are not limited in control problems and also can perform as a full regional approximation controller. In a traditional CMAC, the output value from each hypercube is a constant binary value and it lacks systematic stability analysis for the applications of a CMAC. Some studies have proposed the differential basis function as a replacement for the original constant value of the hypercube's output, in order to obtain the output and input variables' differential message. In 1992, Lane et al. proposed a method that combines a CMAC with a B-spline function, so that a CMAC can learn and store differential information [10]. In 1995, Chiang and Lin proposed a combination of a CMAC and a general basis function (GBF) to develop a new type of cerebellar model articulation controller (CMAC_GBF). The ability of the 
CMAC to learning is improved with differential information [11]. In 1997, Lin and Chiang proved that the learning result for a CMAC_GBF is guaranteed to converge to the best state with the minimum root mean square error [12]. In 2004, Lin and Peng provided a complete stability analysis of a CMAC [13]. Recently, Lin and Li introduced a new Petri fuzzy CMAC [14].

Classification problems are important because, the classification of everything is inherent, in human cognition. Classification uses existing categories to define features, to learn rules by training and to establish the model of the classifier. Therefore, a classifier can be used to determine a new information category [15]. Current classification methods have been widely applied to various problems, such as diseases, bacteria, viruses, RADAR signals and missile detection, etc. Research on medical diagnosis is important and complex. Uncertainty is unavoidable in medical diagnosis [16-18]. The symptoms are one of the uncertainties for an illness. They may or may not occur, or occur as a result of a disease. There is an uncertain relationship between symptoms and disease. Addressing uncertainty allows more accurate decisions to be made. In the past, physicians have given medical diagnoses that mostly rely on past experience, but disease factors have become more diverse. Using techniques for data prediction allows medical practitioners to improve diagnostic accuracy.

\section{The FCMAC Neural Network Architecture}

This paper proposes a generalized fuzzy neural network, which is called a FCMAC. It is an expansion-type fuzzy neural network. A traditional fuzzy neural network can be viewed as a special case of this FCMAC.

\subsection{FCMAC Architecture}

A multi-input, multi-output, FCMAC neural network architecture, is shown in Fig. 1. 


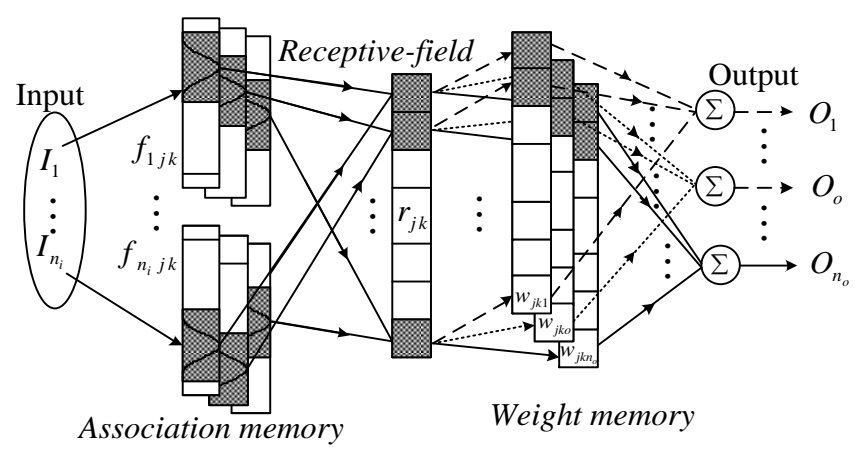

Figure 1

The architecture of a FCMAC

Its fuzzy inference rules are expressed as

$$
\begin{aligned}
& R^{\lambda}: \text { If } I_{1} \text { is } f_{1 j k}, I_{2} \text { is } f_{2 j k}, \cdots, I_{n_{i}} \text { is } f_{n_{i} j k} \text {, then } \\
& o_{o}=w_{j k o} \text { for } j=1,2, \cdots, n_{j}, \quad k=1,2, \cdots, n_{k}, \\
& \lambda=1,2, \cdots, n_{l}, \text { and } o=1,2, \cdots, n_{o}
\end{aligned}
$$

where, $\lambda$ is the $\lambda$-th fuzzy rule, $j$ is the $j$-th layer and $k$ is the $k$-th segment. The difference between this FCMAC and a traditional fuzzy neural network is that it uses the concept of layers and segments, as shown in Fig. 1. If this FCMAC is reduced to only one layer and each segment only contains one neuron, then it is reduced to a traditional fuzzy neural network. Therefore, this FCMAC can be viewed as a generalization of a fuzzy neural network and it learns, recalls and approximates better than a fuzzy neural network. Its signal transmissions and field functions are described below.

a) The Input : For the input space $\boldsymbol{I}=\left[I_{1}, \cdots, I_{i}, \cdots, I_{n_{i}}\right]^{T} \in \mathfrak{R}^{n_{i}}$, every state variable $I_{i}$ is divided into $n_{e}$ discrete regions, called elements or neurons.

b) The Association Memory : Several elements are accumulated in a segment. Each input, $I_{i}$, has $n_{j}$ layers and each layer has $n_{k}$ segments. In this field, each segment uses a Gaussian function as its basis function and is expressed as:

$$
\begin{gathered}
f_{i j k}=\exp \left[\frac{-\left(I_{i}-m_{i j k}\right)^{2}}{v_{i j k}^{2}}\right], \text { for } i=1,2, \cdots, n_{i}, \\
j=1,2, \cdots, n_{j}, \text { and } k=1,2, \cdots, n_{k}
\end{gathered}
$$

where $m_{i j k}$ and $v_{i j k}$ represent the $i$-th input corresponds to the $j$-th layer and the $k$-th segment center value and the variance of the Gaussian function, respectively. 
c) The receptive-field: In this field, the hypercube quantity, $n_{l}$, is equal to the product of $n_{j}$ and $n_{k}$. The receptive-field can be represented as

$r_{j k}=\prod_{i=1}^{n_{i}} f_{i j k}=\prod_{i=1}^{n_{i}} \exp \left[\frac{-\left(I_{i}-m_{i j k}\right)^{2}}{v_{i j k}^{2}}\right]$

for $j=1,2, \cdots, n_{j}$, and $k=1,2, \cdots, n_{k}$

where $r_{j k}$ is the $j$-th layer and the $k$-th segment of the hypercube. Therefore, the multi-dimensional receptive-field basis function can be expressed as a vector:

$\boldsymbol{r}=\left[r_{11}, \cdots, r_{1 n_{k}}, r_{21}, \cdots, r_{2 n_{k}}, \cdots, r_{n_{j} 1}, \cdots, r_{n_{j} n_{k}}\right]^{T} \in \mathfrak{R}^{n_{j} n_{k}}$

d) The weight memory: The memory contents value that corresponds to the hypercube is expressed as:

$$
\begin{gathered}
\boldsymbol{w}_{o}=\left[w_{11 o}, \cdots, w_{1 n_{k} o}, w_{21 o}, \cdots, w_{2 n_{k} o}, \cdots, w_{n_{j} 1 o},\right. \\
\left.\cdots, w_{n_{j} n_{k} o}\right]^{T} \in \mathfrak{R}^{n_{j} n_{k}}, o=1,2, \cdots, n_{0}
\end{gathered}
$$

where $w_{j k o}$ is the $o$-th output that corresponds to value of the weight of the $j$-th layer and the $k$-th segment.

e) The Output : The $o$-th output of FCMAC is represented as:

$y_{o}=\boldsymbol{w}_{o}^{T} \boldsymbol{r}=\sum_{j=1}^{n_{j}} \sum_{k=1}^{n_{k}} w_{j k o} r_{j k}, o=1, \cdots, n_{o}$

\subsection{The Design of the Adaptive FCMAC Classifier}

In this study, this FCMAC neural network is used as an intelligent classifier and is used to identify and classify biomedical bacteria. The literature survey shows that the normalized steepest descent method has fast convergence properties so this method is used to derive the parameters for the learning rules for the adaptive FCMAC classifier. The optimal learning rate for the adjustment rule is also derived so that the FCMAC achieves the fastest convergence. The entire learning algorithm is summarized as follows:

First, we define the energy function:

$$
E(k)=\frac{1}{2} \sum_{o=1}^{n_{o}}\left(d_{o}(k)-y_{o}(k)\right)^{2}=\frac{1}{2} \sum_{o=1}^{n_{o}} e_{o}^{2}(k)
$$

where $e_{o}(k)=d_{o}(k)-y_{o}(k)$ is the output error for the FCMAC classifier and $d_{o}(k)$ and $y_{o}(k)$ are the $o$-th objective and the real output, respectively. Therefore, the FCMAC classifier adjustment rule for weight is described by the following equation: 


$$
\begin{aligned}
\Delta w_{j k o} & =-\eta_{w} \frac{\partial E}{\partial w_{j k o}}=-\eta_{w} \frac{\partial E}{\partial y_{o}} \frac{\partial y_{o}}{\partial w_{j k o}} \\
& =-\eta_{w} \frac{\partial}{\partial y_{o}} \frac{1}{2} \sum_{o=1}^{n_{o}}\left(d_{o}-y_{o}\right)^{2} \frac{\partial}{\partial w_{j k o}} w_{j k o} r_{j k} \\
& =\eta_{w} \sum_{o=1}^{n_{o}} e_{o} r_{j k}
\end{aligned}
$$

where $\eta_{w}$ is the learning rate for weight. The new weight value for the FCMAC classifier is updated as:

$$
w_{j k o}(k+1)=w_{j k o}(k)+\Delta w_{j k o}(k)
$$

The center value and the variance of the receptive-field basis function for the FCMAC is adjusted as:

$$
\begin{aligned}
\Delta m_{i j k} & =-\eta_{m} \sum_{o=1}^{n_{o}} \frac{\partial E(k)}{\partial m_{i j k}}=-\eta_{m} \sum_{o=1}^{n_{o}} \frac{\partial E}{\partial y_{o}} \frac{\partial y_{o}}{\partial r_{j k}} \frac{\partial r_{j k}}{\partial m_{i j k}} \\
& =-\eta_{m} \sum_{o=1}^{n_{0}} \frac{\partial}{\partial y_{o}} \frac{1}{2}\left(d_{o}-y_{o}\right)^{2} \frac{\partial}{\partial r_{j k}} w_{j k o} r_{j k} \frac{\partial}{\partial m_{i j k}} \exp \left(\frac{-\left(I_{i}-m_{i j k}\right)^{2}}{v_{i j k}{ }^{2}}\right) \\
& =-\eta_{m} \sum_{o=1}^{n_{0}}-\left(d_{o}-y_{o}\right) w_{j k o} \exp \left(\frac{-\left(I_{i}-m_{i j k}\right)^{2}}{v_{i j k}{ }^{2}}\right) 2\left(I_{i}-m_{i j k}\right) v_{i j k}^{-2} \\
& =\eta_{m} \sum_{o=1}^{n_{0}} e_{o} w_{j k o} r_{j k} 2\left(I_{i}-m_{i j k}\right) v_{i j k}{ }^{-2} \\
\Delta v_{i j k} & =-\eta_{v} \sum_{o=1}^{n_{o}} \frac{\partial E(k)}{\partial v_{i j k}}=-\eta_{v} \sum_{o=1}^{n_{o}} \frac{\partial E}{\partial y_{o}} \frac{\partial y_{o}}{\partial r_{j k}} \frac{\partial r_{j k}}{\partial v_{i j k}} \\
& =-\eta_{v} \sum_{o=1}^{n_{0}} \frac{\partial}{\partial y_{o}} \frac{1}{2}\left(d_{o}-y_{o}\right)^{2} \frac{\partial}{\partial r_{j k}} w_{j k o} r_{j k} \frac{\partial}{\partial v_{i j k}} \exp \left(\frac{-\left(I_{i}-m_{i j k}\right)^{2}}{v_{i j k}{ }^{2}}\right) \\
& =-\eta_{v} \sum_{o=1}^{n_{0}}-\left(d_{o}-y_{o}\right) w_{j k o} \exp \left(\frac{-\left(I_{i}-m_{i j k}\right)^{2}}{v_{i j k}{ }^{2}}\right) 2\left(I_{i}-m_{i j k}\right)^{2} v_{i j k}{ }^{-3} \\
& =\eta_{v} \sum_{o=1}^{n_{0}} e_{o} w_{j k o} r_{j k} 2\left(I_{i}-m_{i j k}\right)^{2} v_{i j k}{ }^{-3}
\end{aligned}
$$

where $\eta_{m}$ and $\eta_{v}$ are the learning rate for the center value and the variance of the receptive-field basis function, respectively. The new center value and the variance of the receptive-field basis function are updated as:

$$
\begin{aligned}
& m_{i j k}(k+1)=m_{i j k}(k)+\Delta m_{i j k}(k) \\
& v_{i j k}(k+1)=v_{i j k}(k)+\Delta v_{i j k}(k)
\end{aligned}
$$


Although small values for the learning rate guarantee the convergence of FCMCA classifier, the learning process is slow. Large values for the learning rates allow a faster learning speed, but the classifier is less stable. In order to more effectively train the parameters for the FCMAC classifier, the Lyapunov theorem is used to derive variable learning rates that allow the fastest convergence for the output error of the FCMAC classifier.

First, define the gradient operator as $P_{z}(k)=\frac{\partial y_{o}}{\partial z}$, where $z$ is $w, m$ or $v$.

Second, define the Lyapunov function as:

$V(k)=\frac{1}{2} \sum_{o=1}^{n_{o}} e_{o}^{2}(k)$

The variation in $V(k)$ is expressed as:

$\Delta V(k)=V(k+1)-V(k)=\frac{1}{2} \sum_{o=1}^{n_{o}}\left[e_{o}^{2}(k+1)-e_{o}^{2}(k)\right]$

The $o$-th output error for the $k+1$ time is

$e_{o}(k+l)=e_{o}(k)+\Delta e_{o}(k)=e_{o}(k)+\left[\frac{\partial e_{o}(k)}{\partial z}\right]^{T} \Delta z$

Using the chain rule, it can be calculated that

$\frac{\partial e_{o}(k)}{\partial z}=\frac{\partial e_{o}(k)}{\partial y_{o}(k)} \frac{\partial y_{o}(k)}{\partial z}=-P_{z}(k)$

Substituting (17) into (16) gives

$$
\begin{aligned}
e_{o}(k+1) & =e_{o}(k)-\left[P_{z}(k)\right]^{T} \eta_{z} e_{o}(k) P_{z}(k) \\
& =e_{o}(k)\left[1-\eta_{z}\left\|P_{z}(k)\right\|^{2}\right]
\end{aligned}
$$

Substituting (18) into (15) and through simplification:

$\Delta V(k)=\frac{1}{2} \eta_{z} e^{2}(k)\left\|P_{z}(k)\right\|^{2}\left[\eta_{z}\left\|P_{z}(k)\right\|^{2}-2\right]$

The value for $\eta_{z}$ is chosen to be in the $0<\eta_{z}<\frac{2}{\left\|P_{z}(k)\right\|^{2}}$ region, so $V>0$ and $\Delta V<0$ and the Lyapunov stability is guaranteed. When $k \rightarrow \infty$, the convergence of the FCMAC classifier output error $e_{o}(k)$ is guaranteed. The optimal learningrates, $\eta_{z}{ }^{*}$, are selected to achieve the fastest convergence by letting $2 \eta_{z}^{*}\left\|P_{z}(k)\right\|^{2}-2=0$, which results from the derivative of (19) with respect to $\eta_{z}$ and equals to zero. The optimal learning rates are then: 
$\eta_{z}^{*}=\frac{1}{\left\|P_{z}(k)\right\|^{2}}$

In conclusion, the adaptive FCMAC classifier output is defined in (6). Its parameter learning rules are derived by using the normalized steepest descent method. Equations (9), (12) and (13) are used to adjust the output space weights, the center value and the variance of the receptive-field. The optimal learning-rates are defined by (20) and the convergence of FCMAC classifier is guaranteed.

\section{Experimental Results}

As stated earlier, uncertainty is a problem for medical diagnosis. The detection of intestinal bacteria, such as Salmonella or E. coli, allows practitioners to judge whether typhoid or gastrointestinal diseases cause physical discomfort. Using predefined disease feature categories, the detected bacteria are compared with most similar feature to enable a medical diagnosis. The training data (from [19] for Case 1 and from [20] for Case 2) are applied to the FCMAC classifier, presented in Section 2, for learning process with 1000 iterations. When the learning process is completed, the test data are fed into the FCMAC for classification. Then this classifier can classify the samples to allow a medical diagnosis.

\subsection{Medical Sample}

\section{a) Case 1: Bacteria}

The bacterial cells are classified as shown in Fig. 2 [19]. Four bacterial cell characteristic values are shown in Table 1 [19]. F and V represent bacterial feature sets and species, respectively. The information about the bacterial samples includes $F=\{$ Domical shape, Single microscopic shape, Double microscopic shape, Flagellum $\}$ and V $=\{$ Bacillus coli, Shigella, Salmonella, Klebsiella $\}$. The samples for classification are shown in Table 2 [19]. 


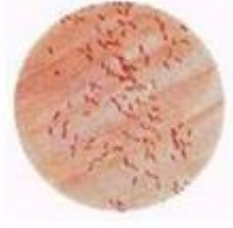

Bacillus coli

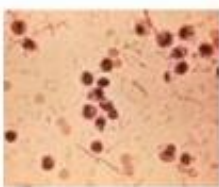

Shigella

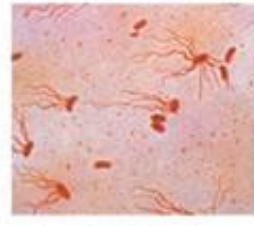

Salmonell

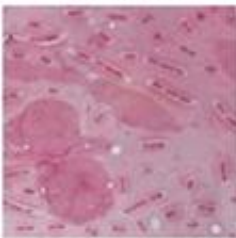

Klebsiella

Figure 2

Different types of bacterial cell samples [19]

Table 1

The features' values in the studied bacteria classes (base patterns)

\begin{tabular}{ccccc}
\hline \multirow{2}{*}{ Bacteria } & \multicolumn{5}{c}{ Features } \\
\cline { 2 - 5 } & F1 & F2 & F3 & F4 \\
\hline V1 & $\langle 0.850,0.050\rangle$ & $\langle 0.870,0.010\rangle$ & $\langle 0.020,0.970\rangle$ & $\langle 0.920,0.060\rangle$ \\
\hline V2 & $\langle 0.830,0.080\rangle$ & $\langle 0.920,0.050\rangle$ & $\langle 0.050,0.920\rangle$ & $\langle 0.080,0.910\rangle$ \\
\hline V3 & $\langle 0.790,0.120\rangle$ & $\langle 0.780,0.110\rangle$ & $\langle 0.110,0.850\rangle$ & $\langle 0.870,0.010\rangle$ \\
\hline V4 & $\langle 0.820,0.150\rangle$ & $\langle 0.720,0.150\rangle$ & $\langle 0.220,0.750\rangle$ & $\langle 0.120,0.850\rangle$ \\
\hline
\end{tabular}

Table 2

The features' values of the samples detection

\begin{tabular}{ccccc}
\hline \multirow{2}{*}{ Samples } & \multicolumn{5}{c}{ Features } \\
\cline { 2 - 5 } & F1 & F2 & F3 & F4 \\
\hline S1 & $\langle 0.873,0.133\rangle$ & $\langle 0.718,0.159\rangle$ & $\langle 0.064,0.897\rangle$ & $\langle 0.021,0.806\rangle$ \\
\hline S2 & $\langle 0.911,0.029\rangle$ & $\langle 0.831,0.031\rangle$ & $\langle 0.028,0.894\rangle$ & $\langle 0.952,0.036\rangle$ \\
\hline S3 & $\langle 0.929,0.037\rangle$ & $\langle 0.812,0.033\rangle$ & $\langle 0.021,0.926\rangle$ & $\langle 0.054,0.922\rangle$ \\
\hline S4 & $\langle 0.815,0.091\rangle$ & $\langle 0.949,0.048\rangle$ & $\langle 0.020,0.880\rangle$ & $\langle 0.833,0.042\rangle$ \\
\hline S5 & $\langle 0.864,0.020\rangle$ & $\langle 0.610,0.230\rangle$ & $\langle 0.243,0.624\rangle$ & $\langle 0.000,0.964\rangle$ \\
\hline S6 & $\langle 0.905,0.016\rangle$ & $\langle 0.878,0.015\rangle$ & $\langle 0.072,0.917\rangle$ & $\langle 0.789,0.114\rangle$ \\
\hline
\end{tabular}

\section{b) Case 2: Disease}

The symptomatic characteristics of the target sample are shown in Table 3 [20]. There are five diseases: viral fever, malaria, typhoid, stomach problems and chest problems. Each disease has five features: temperature, headache, stomach pain, coughs and chest pain. Four disease samples classified are shown in Table 4 [20]. 
Table 3

Symptoms feature of five diseases

\begin{tabular}{cccccc}
\hline & Temperature & Headache & Stomach pain & Cough & Chest pain \\
\hline Viral fever & $(0.4,0.0,0.6)$ & $(0.3,0.5,0.2)$ & $(0.1,0.7,0.2)$ & $(0.4,0.3,0.3)$ & $(0.1,0.7,0.2)$ \\
\hline Malaria & $(0.7,0.0,0.3)$ & $(0.2,0.6,0.2)$ & $(0.0,0.9,0.1)$ & $(0.7,0.0,0.3)$ & $(0.1,0.8,0.1)$ \\
\hline Typhoid & $(0.3,0.3,0.4)$ & $(0.6,0.1,0.3)$ & $(0.2,0.7,0.1)$ & $(0.2,0.6,0.2)$ & $(0.1,0.9,0.0)$ \\
\hline $\begin{array}{c}\text { Stomach } \\
\text { problem }\end{array}$ & $(0.1,0.7,0.2)$ & $(0.2,0.4,0.4)$ & $(0.8,0.0,0.2)$ & $(0.2,0.7,0.1)$ & $(0.5,0.7,0.1)$ \\
\hline $\begin{array}{c}\text { Chest } \\
\text { problem }\end{array}$ & $(0.1,0.8,0.1)$ & $(0.0,0.8,0.0)$ & $(0.2,0.8,0.0)$ & $(0.2,0.8,0.0)$ & $(0.8,0.1,0.1)$ \\
\hline
\end{tabular}

Table 4

Symptoms feature of four people disease samples

\begin{tabular}{cccccc}
\hline & Temperature & Headache & Stomach pain & Cough & Chest pain \\
\hline $\mathrm{Al}$ & $(0.8,0.1,0.1)$ & $(0.6,0.1,0.3)$ & $(0.2,0.8,0.0)$ & $(0.6,0.1,0.3)$ & $(0.1,0.6,0.3)$ \\
\hline Bob & $(0.0,0.8,0.2)$ & $(0.4,0.4,0.2)$ & $(0.6,0.1,0.3)$ & $(0.1,0.7,0.2)$ & $(0.1,0.8,0.1)$ \\
\hline Joe & $(0.8,0.1,0.1)$ & $(0.8,0.1,0.1)$ & $(0.0,0.6,0.4)$ & $(0.2,0.7,0.1)$ & $(0.0,0.5,0.5)$ \\
\hline Ted & $(0.6,0.1,0.3)$ & $(0.5,0.4,0.1)$ & $(0.3,0.4,0.3)$ & $(0.7,0.2,0.1)$ & $(0.3,0.4,0.3)$ \\
\hline
\end{tabular}

\subsection{Results}

\section{a) Case 1: Bacteria}

The concept of clustering is used to determine the similarity between each known bacteria and unknown sample features. Four information features of known bacteria and unknown samples are used for a pairwise comparison. For example, each known bacterial first feature value combination of F1 and F2 is compared with the same feature value combination for each unknown sample and each known bacterial first feature value combination of F1 and F3 is compared with the same feature value combination for each unknown sample. The six reference figures, Fig. 3 to Fig. 8, show the results.

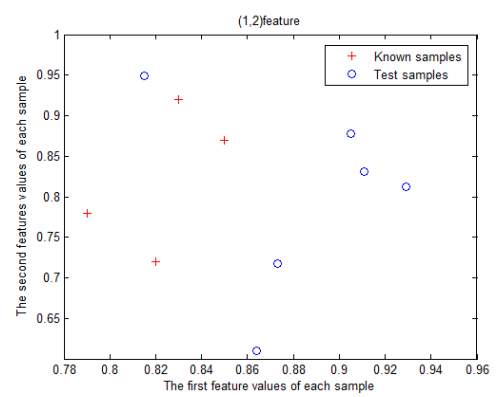

Figure 3

$(1,2)$ feature distribution 


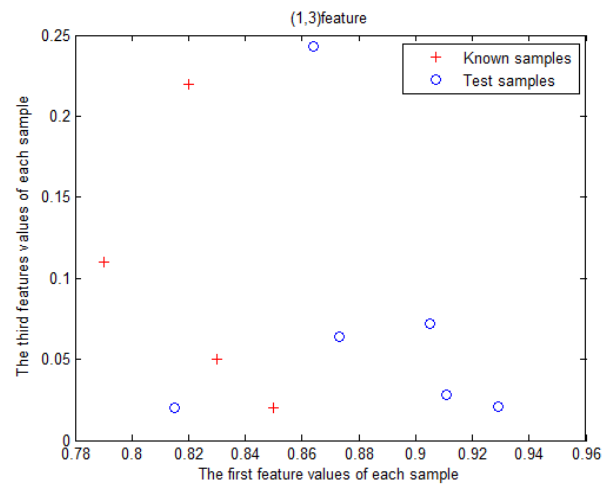

Figure 4

$(1,3)$ feature distribution

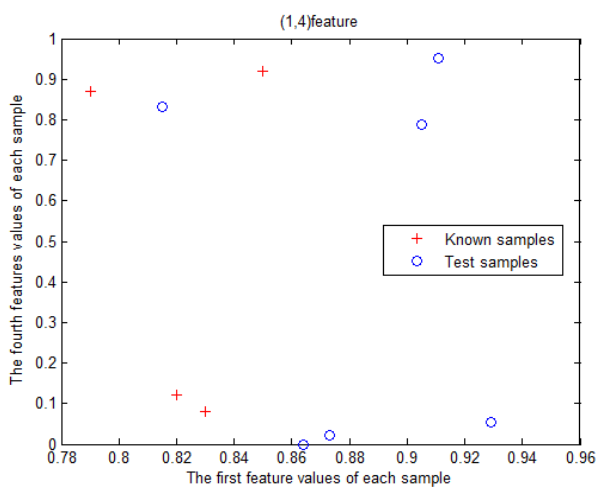

Figure 5

$(1,4)$ feature distribution

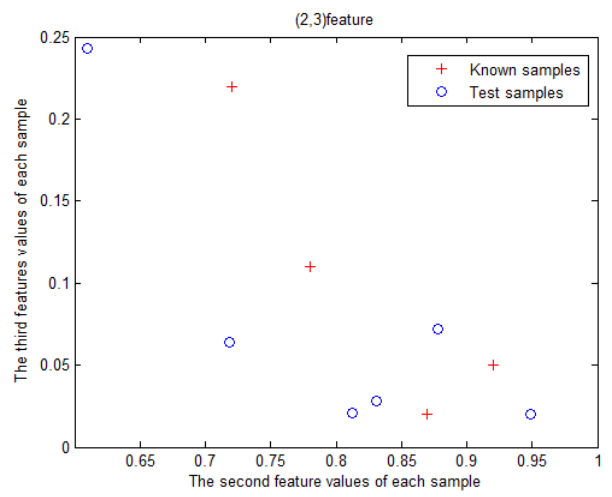

Figure 6

$(2,3)$ feature distribution 


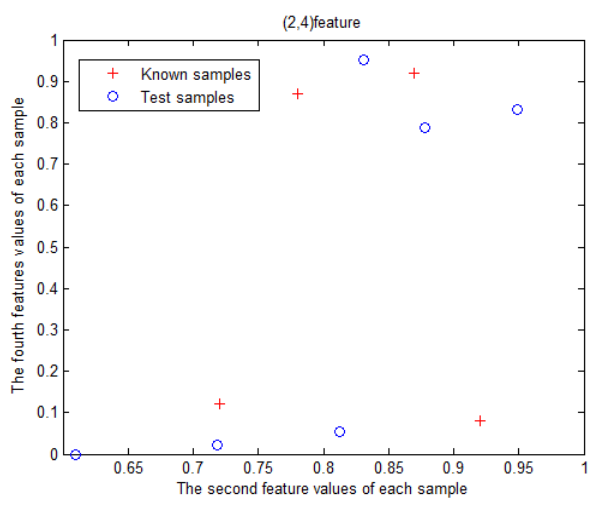

Figure 7

$(2,4)$ feature distribution

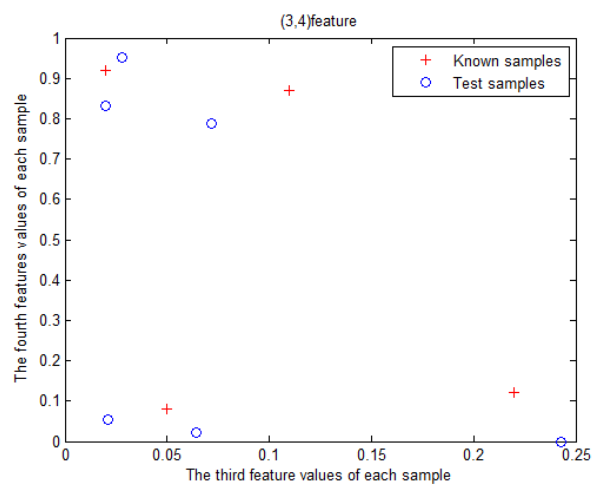

Figure 8

$(3,4)$ feature distribution

The proposed FCMAC classifier identifies the bacterial samples.

The bacterial sample data is

$$
m=\left[\begin{array}{llll}
0.85 & 0.87 & 0.02 & 0.92 \\
0.83 & 0.92 & 0.05 & 0.08 \\
0.79 & 0.78 & 0.11 & 0.87 \\
0.82 & 0.72 & 0.22 & 0.12
\end{array}\right]
$$

Table 5 shows the classification results for the intuitive fuzzy set (IFS) method [20] and the proposed FCMAC. The correct recognition rate shows that the proposed FCMAC classifier performs better than the IFS classifier. 
Table 5

IFS and FCMAC classification results

\begin{tabular}{ccc}
\hline Identification method & Correct recognition rate & Error recognition rate \\
\hline IFS & $95.27 \%$ & $4.73 \%$ \\
\hline FCMAC & $97.55 \%$ & $2.45 \%$ \\
\hline
\end{tabular}

b) Case 2: Disease

Using Table 3 and Table 4, the ten reference figures, Fig. 9 to Fig. 18, show the degree of difficulty of classification. Table 6 shows that the FCMAC classifier can achieve exact classification and produce good recognition results. The proposed classifier will allow medical practitioners to improve diagnostic accuracy.

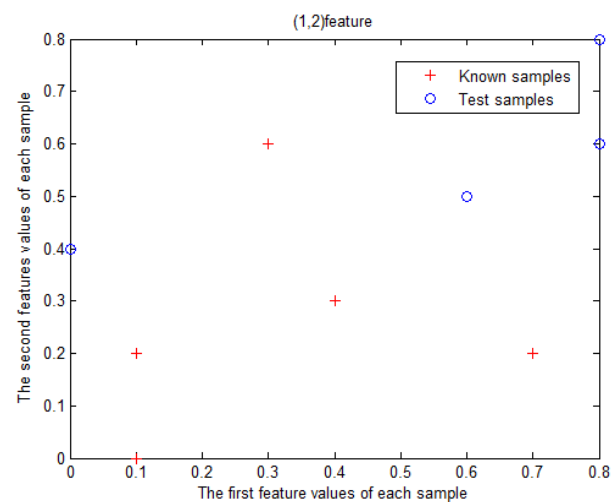

Figure 9

$(1,2)$ feature distribution

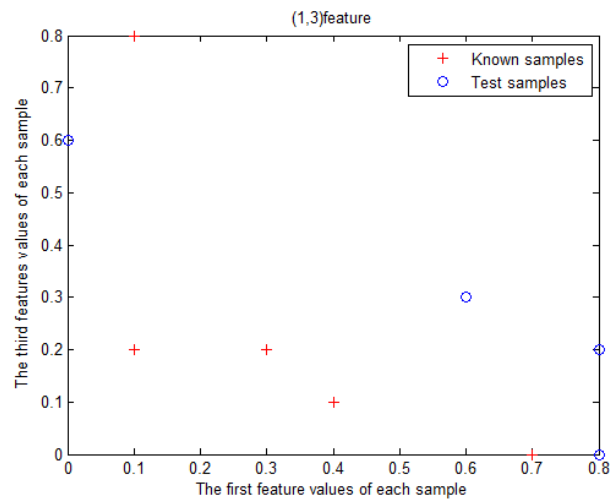

Figure 10

$(1,3)$ feature distribution 


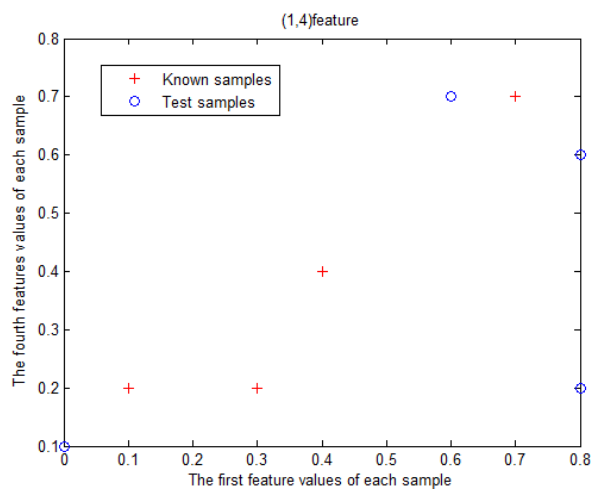

Figure 11

$(1,4)$ feature distribution

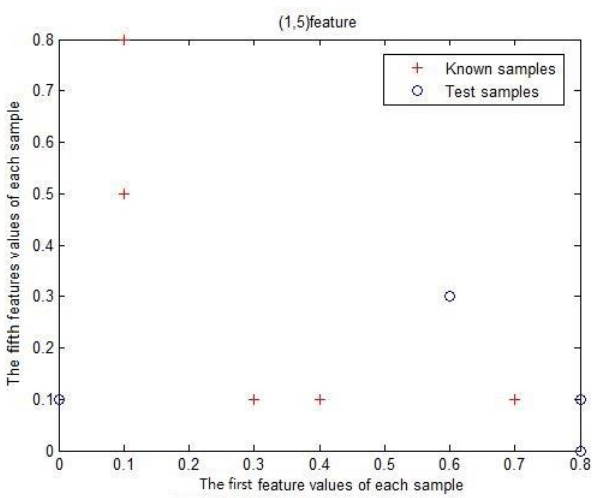

Figure 12

$(1,5)$ feature distribution

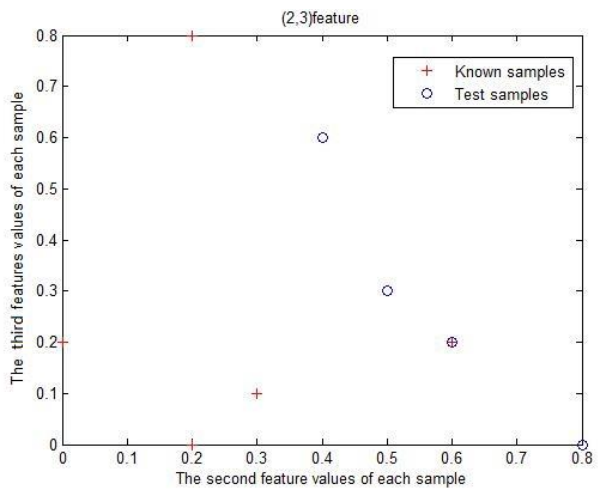

Figure 13

$(2,3)$ feature distribution 


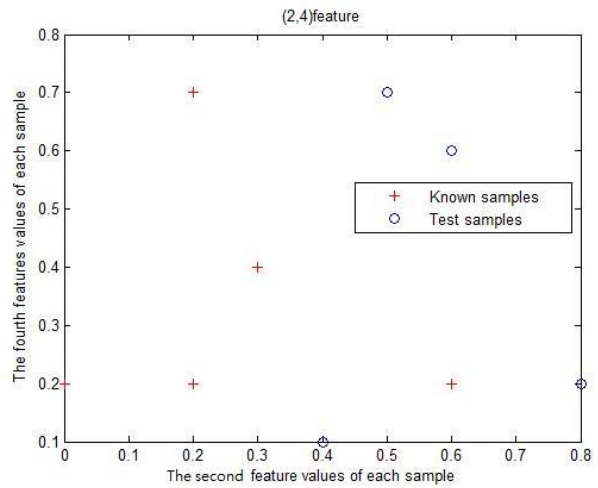

Figure 14

$(2,4)$ feature distribution

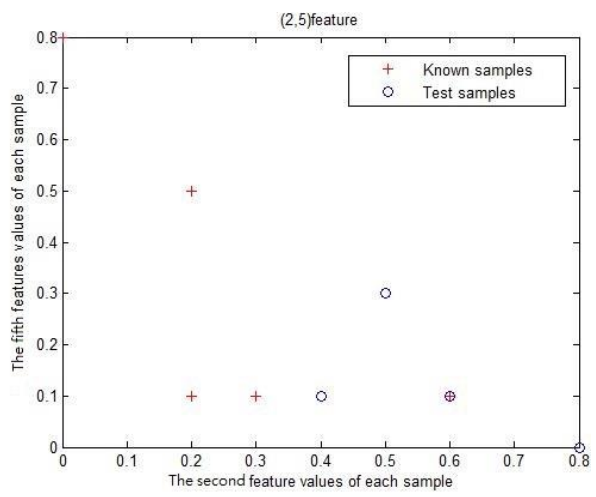

Figure 15

$(2,5)$ feature distribution

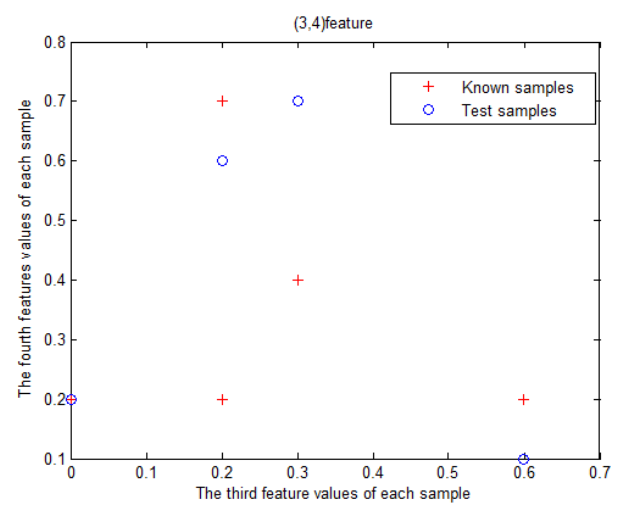

Figure 16

$(3,4)$ feature distribution 


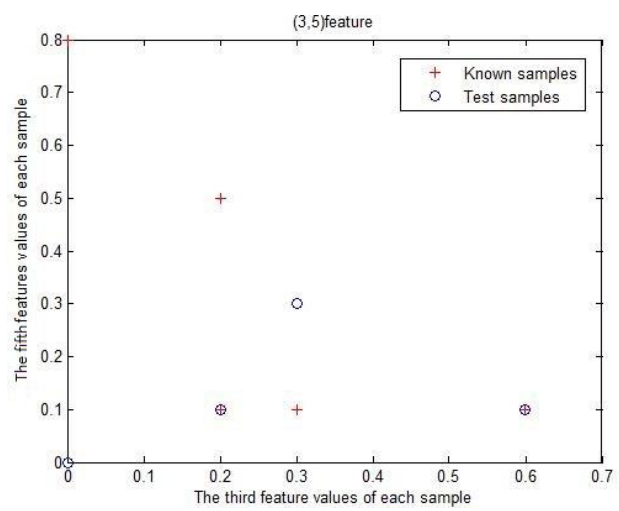

Figure 17

$(3,5)$ feature distribution

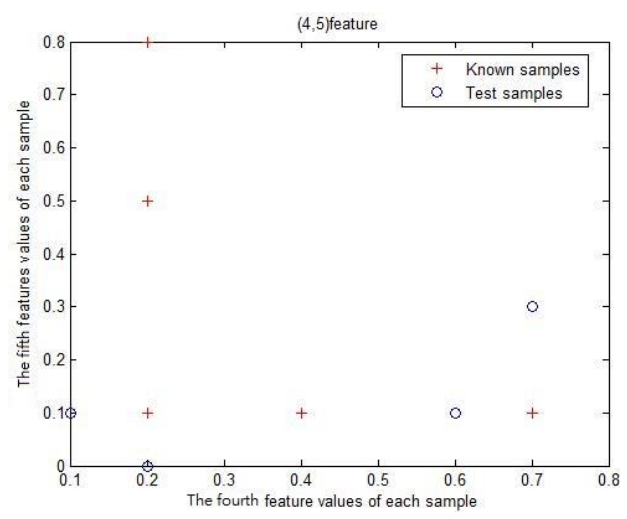

Figure 18

$(4,5)$ feature distribution

Table 6

FCMAC classification results

\begin{tabular}{cccccc}
\hline & Viral fever & Malaria & Typhoid & Stomach problem & Chest problem \\
\hline $\mathrm{Al}$ & $\mathbf{1}$ & 0 & 0 & 0 & 0 \\
\hline Bob & 0 & 0 & 0 & $\mathbf{1}$ & 0 \\
\hline Joe & $\mathbf{1}$ & 0 & 0 & 0 & 0 \\
\hline Ted & $\mathbf{1}$ & 0 & 0 & 0 & 0 \\
\hline
\end{tabular}

\section{Conclusions}

This paper proposes a generalized fuzzy neural network, called a fuzzy CMAC (FCMAC), which combines fuzzy rules and CMAC. This network is used as an adaptive FCMAC classifier that allows stable classification and a fast convergence. 
The developed network is an expansion-type fuzzy neural network. A traditional fuzzy neural network can be viewed as a special case of this network.

This expansion-type fuzzy neural network allows better generalization, learning and approximation than a traditional fuzzy neural network so it greatly increases the effectiveness of a neural network classifier. The Lyapunov stability theory is used to develop the learning rule, for classifier parameters, in order to allow online self-adjustment. Therefore, the convergence of the design classifier is guaranteed.

This study successfully extends the application of the proposed adaptive FCMAC neural network to the classification of medical samples. The effectiveness of the expansion-type FCMAC neural network is verified by the simulation results.

\section{Acknowledgement}

The authors appreciate the financial support in part from the Nation Science Council of Republic of China under grant NSC 101-2221-E-155-026-MY3.

\section{References}

[1] S. S. Ge, C. C. Hang, and T. Zhang, "Adaptive Neural Network Control of Nonlinear Systems by State and Output Feedback," IEEE Trans. Syst., Man, Cybern., B, Vol. 29, No. 6, pp. 818-828, 1999

[2] S. K. Oh, W. D. Kim, W. Pedrycz and B. J. Park, "Polynomial-based Radial Basis Function Neural Networks (P-RBF NNs) Realized with the Aid of Particle Swarm Optimization," Fuzzy Sets and Systems, Vol. 163, No. 1, pp. 54-77, 2011

[3] C. M. Lin, A. B. Ting, C. F. Hsu and C. M. Chung, "Adaptive Control for MIMO Uncertain Nonlinear Systems Using Recurrent Wavelet Neural Network," International Journal of Neural Systems, Vol. 22, No. 1, pp. $37-$ 50,2012

[4] C. F. Hsu, C. M. Lin and R. G. Yeh, "Supervisory Adaptive Dynamic RBFbased Neural-Fuzzy Control System Design for Unknown Nonlinear Systems," Applied Soft Computing, Vol. 13, No. 4, pp. 1620-1626, 2013

[5] D. Marr, "A Theory of Cerebellar Cortex," J. Physiol., Vol. 202, pp. 437470, 1969

[6] J. S. Albus, "A New Approach to Manipulator Control: the Cerebellar Model Articulation Controller (CMAC)," ASME, J. Dynam., Syst., Meas., Contr., Vol. 97, No. 3, pp. 220-227, 1975

[7] J. S. Albus, "Data Storage in the Cerebellar Model Articulation Controller (CMAC)," ASME, J. Dynam., Syst., Meas., Contr., Vol. 97, No. 3, pp. 228233, 1975

[8] W. T. Miller, F. H. Glanz, and L. G. Kraft, "CMAC: an Associative Neural Network Alternative to Backpropagation," Proc. of the IEEE, Vol. 78, No. 10, pp. 1561-1567, 1990 
[9] R. J. Wai, C. M. Lin, and Y. F. Peng, "Robust CMAC Neural Network Control for LLCC Resonant Driving Linear Piezoelectric Ceramic Motor," IEE Proc., Cont. Theory, Applic., Vol. 150, No. 3, pp. 221-232, 2003

[10] S. H. Lane, D. A. Handelman, J. J. Gelfand, "Theory and Development of Higher-Order CMAC Neural Network”, IEEE Control Systems Magazine, Vol. 12, No. 2, pp. 23-30, 1992

[11] C. T. Chang and C. S. Lin, "CMAC with General Basis Functions," Neural Network, Vol. 9, No. 7, pp. 1199-1211, 1996

[12] C. S. Lin and C. T. Chiang, "Learning Convergence of CMAC Technique," IEEE Trans. Neural Networks, Vol. 8, No. 6, pp. 1281-1292, 1997

[13] C. M. Lin and Y. F. Peng, "Adaptive CMAC-based Supervisory Control for Uncertain Nonlinear Systems," IEEE Trans. System, Man, and Cybernetics Part B, Vol. 34, No. 2, pp. 1248-1260, 2004

[14] C. M. Lin and H. Y. Li, "Dynamic Petri Fuzzy Cerebellar Model Articulation Control System Design for Magnetic Levitation System," IEEE Control Systems Technology, Vol. 23, No. 2, pp. 693-699, 2015

[15] G. Gosztolya and L. Szilagyi, "Application of Fuzzy and Possibilistic cmeans Clustering Models in Blind Speaker Clustering," Acta Polytechnica Hungarica, Vol. 12, No. 7, pp. 41-56, 2015

[16] J. Zheng, W. Zhuang,, N. Yan, G. Kou, H. Peng, C. McNally, D. Erichsen, A. Cheloha, S. Herek, C. Shi, Y. Shi, " Classification of HIV-I-mediated Neuronal Dendritic and Synaptic Damage using Multiple Criteria Linear Programming, Neuroinformatics, Vol. 2, No. 3, pp. 303-326, 2004

[17] E. Straszecka, "Combining Uncertainty and Imprecision in Models of Medical Diagnosis," Information Sciences, Vol. 176, No. 20, pp. 30263059, 2006

[18] J. W. Han and M. Kamber, Data mining: concepts and techniques. Morgan, San Mateo, CA, 2006.

[19] V. Khatibi and G. A Montazer, "Intuitionistic Fuzzy Set vs. Fuzzy Set Application in Medical Pattern Recognition," Artificial Intelligence in Medicine, Vol. 47, pp. 43- 52, 2009

[20] K. C. Hung, "Medical Pattern Recognition: Applying an Improved Intuitionistic Fuzzy Cross-Entropy Approach," Advances in Fuzzy Systems, Published on line, Vol. 2012, Article ID 863549, 6 pages, 2012 